\title{
Cinacalcet Treatment Significantly Improves All-Cause and Cardiovascular Survival in Dialysis Patients: Results from a Meta-Analysis
}

\author{
Yuan Zu Xiangxue Lu Jinghong Song Ling Yu Han Li Shixiang Wang \\ Department of Blood Purification, Beijing Chao-Yang Hospital, Capital Medical University, \\ Beijing, China
}

\section{Keywords}

Cinacalcet $\cdot$ All-cause mortality $\cdot$ Cardiovascular mortality $\cdot$ Dialysis

\begin{abstract}
Objective: To assess the long-term effects including all-cause mortality, cardiovascular mortality, and fracture incidence, of cinacalcet on secondary hyperparathyroidism (SHPT) in patients on dialysis. Methods: PubMed, Embase, and the Cochrane Central Register of Controlled Trials were searched from their inception to October 2018. Randomized controlled trials (RCTs) and cohort design prospective observational studies assessing cinacalcet for the treatment of SHPT in dialysis patients were included. Data extraction was independently completed by 2 authors who determined the methodological quality of the studies and extracted data in duplicate. Study-specific risk estimates were tested by using a fixed effects model. Results: A total of 14 articles with 38,219 participants were included, of which 10 RCTs with 7,471 participants and 4 prospective observational studies with 30,748 participants fulfilled the eligibility criteria. Compared with no cinacalcet, cinacalcet administration reduced allcause mortality (relative risk [RR] 0.91, 95\% Cl 0.89-0.94, $p<0.001$ ) and cardiovascular mortality (RR $0.92,95 \% \mathrm{Cl} 0.89-0.95, p<0.001)$, but it did not significantly reduce the incidence of fractures (RR $0.93,95 \% \mathrm{Cl} 0.87-1.00, p=0.05$ ). Conclusions: The results of this meta-analysis indicated that the treatment of SHPT with cinacalcet may in fact reduce all-cause mortality and cardiovascular mortality among patients receiving maintenance dialysis.
\end{abstract}

\footnotetext{
(c) 2019 The Author(s)

Published by S. Karger AG, Basel
}

Y.Z. and X.L. contributed equally to this work. 


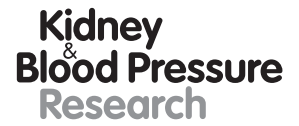

Research

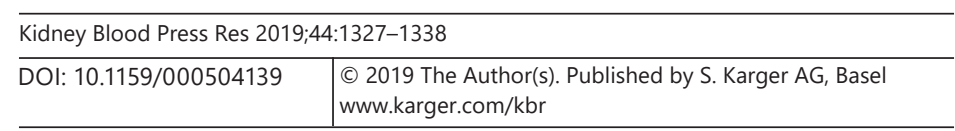

Zu et al.: Cinacalcet Treatment Improves All-Cause and Cardiovascular Survival in Dialysis Patients

Chronic kidney disease (CKD) is increasingly common in developing and developed countries, and it has become a global public health issue [1]. CKD-mineral and bone disorder (CKD-MBD) involves abnormal serum concentrations of calcium, phosphorus, parathyroid hormone (PTH), and vitamin D. Correcting these abnormalities is important because they are associated with cardiovascular disease and mortality, which occur relatively frequently in hemodialysis patients [2].

Cinacalcet, a second-generation calcimimetic agent, is a positive allosteric modulator of the calcium-sensing receptor that increases its sensitivity to extracellular calcium by lowering the threshold for activation by extracellular calcium ions [3, 4]. This mechanism lowers PTH synthesis and secretion. Treatment with cinacalcet can reduce serum calcium, often accompanied by a reduction in serum phosphate, as shown in multiple randomized, controlled trials. Treatment with cinacalcet increases the risk of adverse events, such as hypocalcemia, nausea, vomiting, and diarrhea. Several meta-reviews have also confirmed the effects and adverse effects of cinacalcet on secondary hyperparathyroidism (SHPT) in CKD patients [5, 6]. However, hypocalcemia-induced serious adverse events were very rare.

By slowing the progression of vascular calcification mediated by hypercalcemia and hyperphosphatemia, cinacalcet might prevent death, particularly death due to cardiovascular disease $[7,8]$. Some randomized controlled trials (RCTs) found that cinacalcet significantly reduced the likelihood of parathyroidectomy, fracture, and cardiovascular hospitalization; improved physical function; and diminished pain. However, the effect on all-cause and cardiovascular mortality was not demonstrated $[9,10]$. RCTs with clinical outcomes are considered the gold standard for regulatory approval. However, by design, it is difficult to follow longterm clinical outcomes in RCTs [11]. RCT experiments are difficult to follow-up for enough time to observe the risk of death. Therefore, high-quality observational studies are required for clinical decision-making. Some high-quality observational studies found that all-cause and cardiovascular mortality rates were significantly lower for those treated with cinacalcet than for those that were not $[12,13]$. Relevant meta-analysis results are also inconsistent. Few meta-analyses take into account the observational experiments. We performed this metaanalysis including RCTs and high-quality observational studies to provide further evidence regarding the effects of cinacalcet on clinical outcomes in hemodialysis patients.

\section{Methods}

\section{Data Sources and Searches}

We searched PubMed, Embase, and the Cochrane Library from their inception to October 2018, and we systematically identified clinical studies evaluating the effects of cinacalcet in dialysis patients. No language restriction was applied. The search strategy included terms for cinacalcet, SHPT, and hemodialysis. We also scanned the cited references of retrieved articles and all prior systemic reviews to identify any additional relevant studies.

\section{Eligibility Criteria}

We included studies that evaluated the effectiveness and safety of cinacalcet for the treatment of SHPT in dialysis patients. A published article was included if (1) it was an RCT or a casecontrol or cohort design prospective observational study, (2) the study evaluated the administration of cinacalcet alone or in combination with a vitamin D compound or phosphate binder versus the administration of placebo in combination with standard treatment or standard treatment alone, and (3) the end point events (all-cause or cardiovascular mortality and fracture incidence) and their corresponding hazard ratio (HR) or relative risk (RR) and its $95 \% \mathrm{CI}$ were reported or could be calculated from data reported. If publications were duplicated or if the 
Table 1. Methodological quality of cohort studies included in the meta-analysis

\begin{tabular}{|c|c|c|c|c|c|c|c|c|c|c|}
\hline Cohort study & $\begin{array}{l}\text { Representative- } \\
\text { ness of the } \\
\text { exposed cohort }\end{array}$ & $\begin{array}{l}\text { Selection } \\
\text { of the } \\
\text { unexposed } \\
\text { cohort }\end{array}$ & $\begin{array}{l}\text { Ascertainment } \\
\text { of exposure }\end{array}$ & $\begin{array}{l}\text { Outcome } \\
\text { of interest } \\
\text { not present } \\
\text { at start } \\
\text { of study }\end{array}$ & $\begin{array}{l}\text { Control for } \\
\text { important } \\
\text { factor or } \\
\text { additional } \\
\text { factor }\end{array}$ & $\begin{array}{l}\text { Outcome } \\
\text { assessment }\end{array}$ & $\begin{array}{l}\text { Follow-up } \\
\text { long enough } \\
\text { form outcomes } \\
\text { to occur }{ }^{1}\end{array}$ & $\begin{array}{l}\text { Adequacy } \\
\text { of follow-up } \\
\text { of cohorts }\end{array}$ & $\begin{array}{l}\text { Data analysis } \\
\text { using energy } \\
\text { adjusted residual } \\
\text { model or nutrient } \\
\text { density model }\end{array}$ & $\begin{array}{l}\text { Total } \\
\text { quality } \\
\text { scores }\end{array}$ \\
\hline Block et al. [25], 2010 & Yes & Yes & Yes & Yes & Yes & Yes & Yes & No & No & 8 \\
\hline Gillespie et al. [13], 2015 & Yes & Yes & Yes & Yes & Yes & Yes & Yes & No & No & 8 \\
\hline Akizawa et al. [26], 2016 & Yes & Yes & Yes & Yes & Yes & Yes & Yes & No & No & 8 \\
\hline
\end{tabular}

${ }^{1}$ A cohort study with a follow-up rate more than $75 \%$ was assigned one star. ${ }^{2}$ A cohort study with a follow-up time more than 8 years was assigned one star.

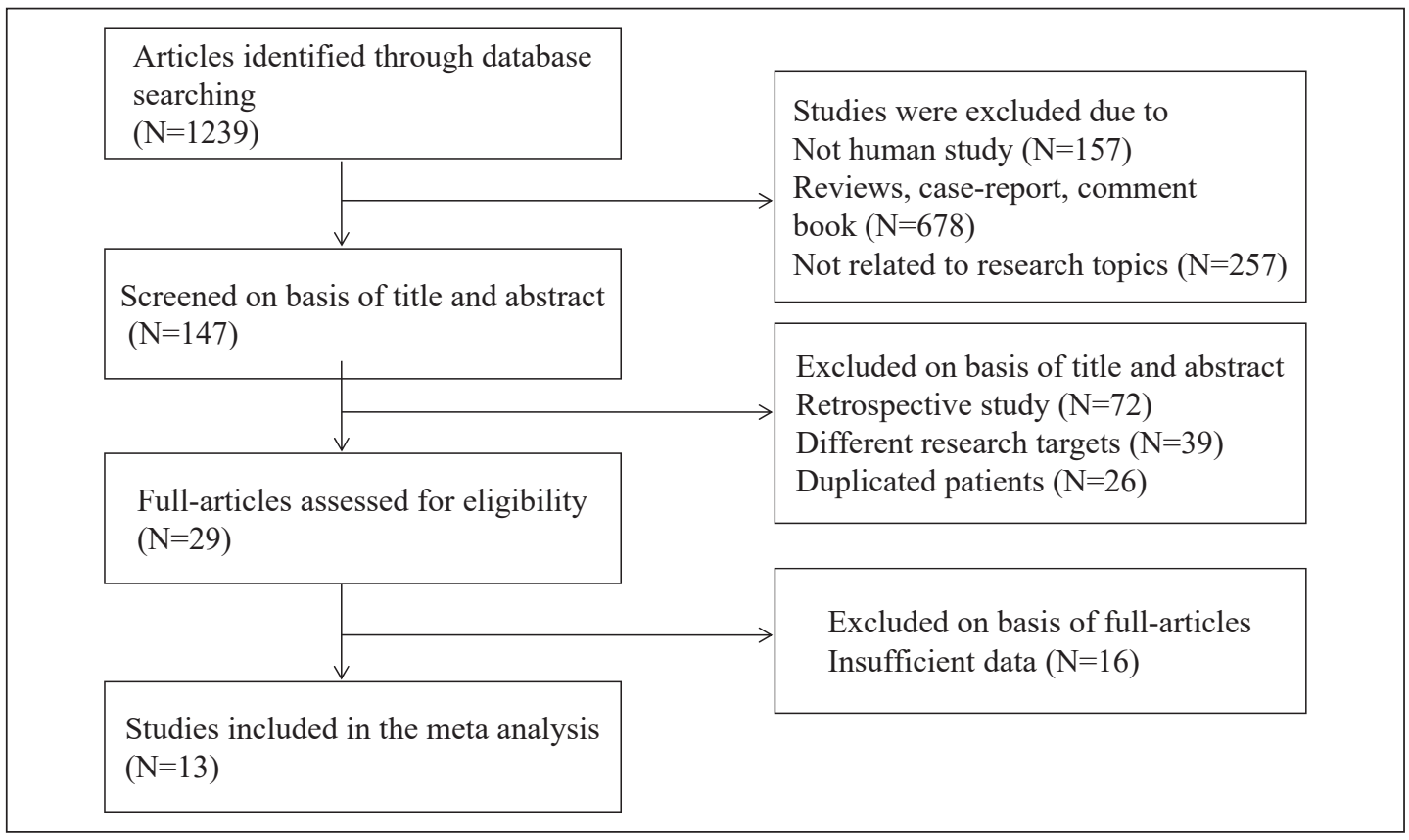

Fig. 1. Flow diagram of the study selection.

articles were from the same study population, the most recent publication was included. Excluded from this analysis were studies that were retrospective studies, those that included nondialysis patients, or those for which the HR or RR of the end point events could not be obtained.

Two authors independently screened the abstracts and full texts of all potentially eligible articles; disagreements between reviewers were resolved by a third reviewer. The risk of bias in RCTs was assessed using the Cochrane Collaboration's tool [14]. To assess the prospective observational study quality, a 9-star system based on the Newcastle-Ottawa Scale was used, in which a study was judged on 3 broad perspectives: the selection of the study groups, the comparability of the groups, and the ascertainment of either the exposure or the outcome of interest for case-control or prospective studies. A high-quality study was defined as a study that was awarded $\geq 7$ stars (Table 1). Our meta-analysis of RCTs and prospective observational studies were performed according to both Preferred Reporting Items for Systematic Reviews [15] and Meta-analysis Of Observational Studies in Epidemiology recommendations [16].

Data synthesis was conducted and RevMan software (version 5.3; Cochrane Collaboration, Copenhagen, Denmark) was used for statistical analysis. To compute a summary RR with its 95\% CI, we used the study-specific, most adjusted RR or HR (for all-cause mortality, cardiovascular mortality, and fracture incidence) and its 95\% CI in all analyses. logHR or 
Zu et al.: Cinacalcet Treatment Improves All-Cause and Cardiovascular Survival in Dialysis Patients

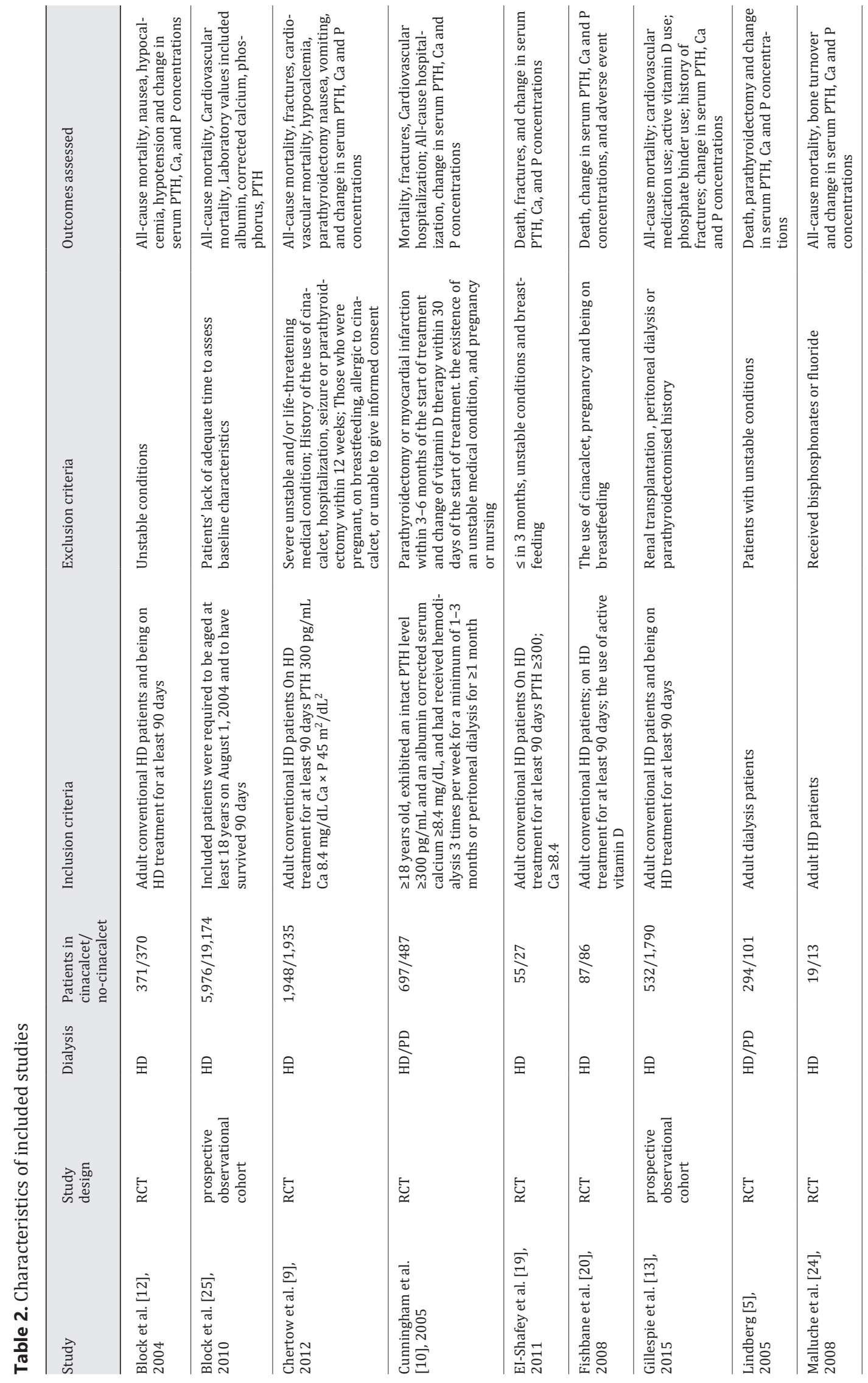


Kidney

Blood Pressure

Research

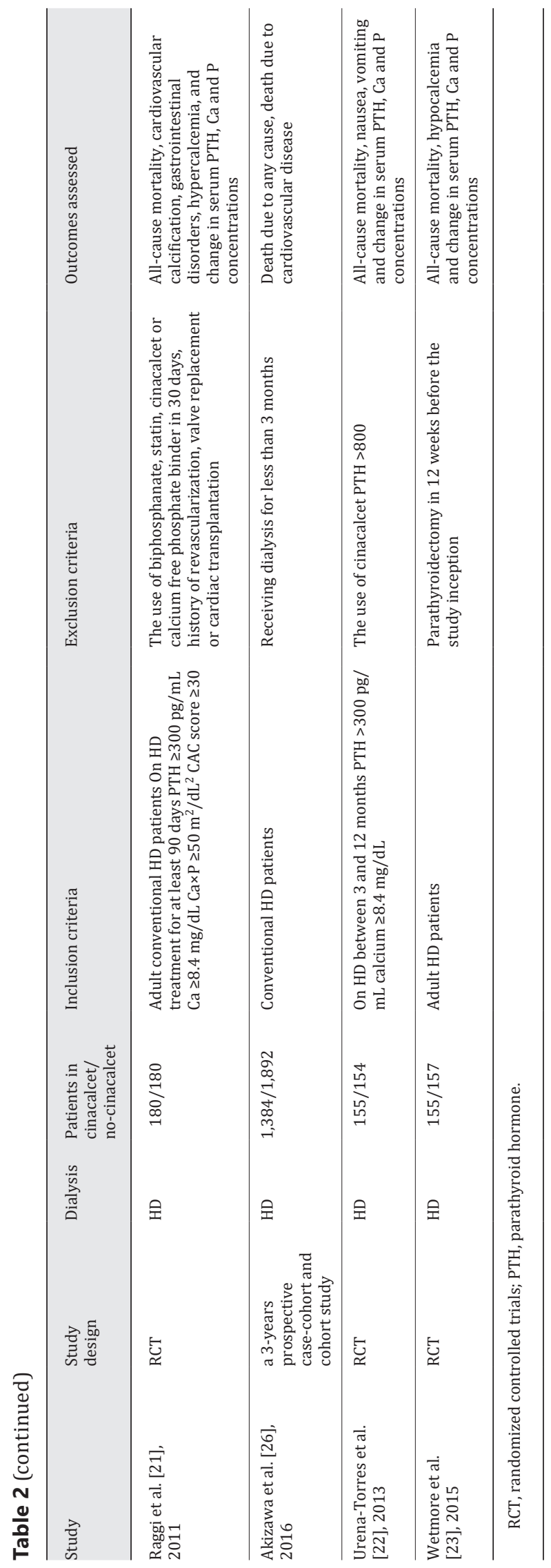

\begin{tabular}{l|l}
\hline Kidney Blood Press Res 2019:44:1327-1338 \\
\hline DOI: 10.1159/000504139 & $\begin{array}{l}\text { ○ 2019 The Author(s). Published by S. Karger AG, Basel } \\
\text { www.karger.com/kbr }\end{array}$ \\
\hline
\end{tabular}

Zu et al.: Cinacalcet Treatment Improves All-Cause and Cardiovascular Survival in Dialysis Patients 
Zu et al.: Cinacalcet Treatment Improves All-Cause and Cardiovascular Survival in Dialysis Patients

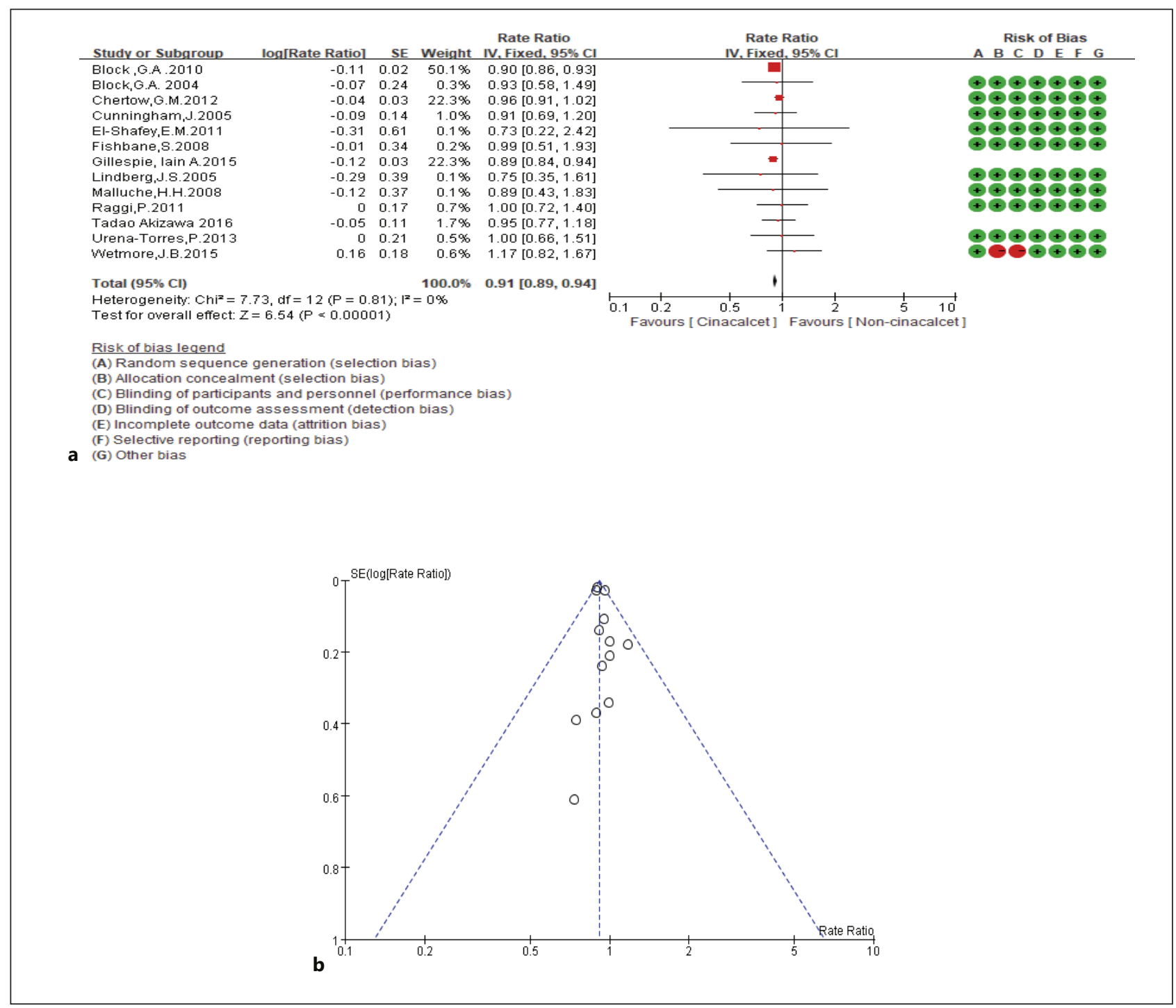

Fig. 2. a Forest plot of all-cause mortality in the cinacalcet group versus the no-cinacalcet control group. b Funnel plot to assess publication bias in effect estimates in all-cause mortality.

$\operatorname{logRR}$ and SElogHR or SElogOR needed to be entered. SElogHR $=(\log U \mathrm{CI}-\log \mathrm{LCI}) / 3.92$. A $p$ value $<0.05$ was considered statistically significant. We examined heterogeneity in results across studies using the Cochran $\mathrm{Q}$ and $I^{2}$ statistics. The null hypothesis that the studies are homogeneous was rejected if the $p$ value for heterogeneity was $<0.10$ or if $I^{2}$ was $>50 \%[17$, 18]. We employed a funnel plot to assess publication bias and a fixed effects model (the inverse variance method) to estimate study-specific risk.

\section{Results}

Characteristics and quality of the studies. A total of 38,219 potentially relevant citations were identified and screened. Thirteen articles were retrieved or detailed, of which 10 RCTs $[6,9,10,12,19-24]$ with 7,471 participants and 3 prospective observational studies $[13,25$, 


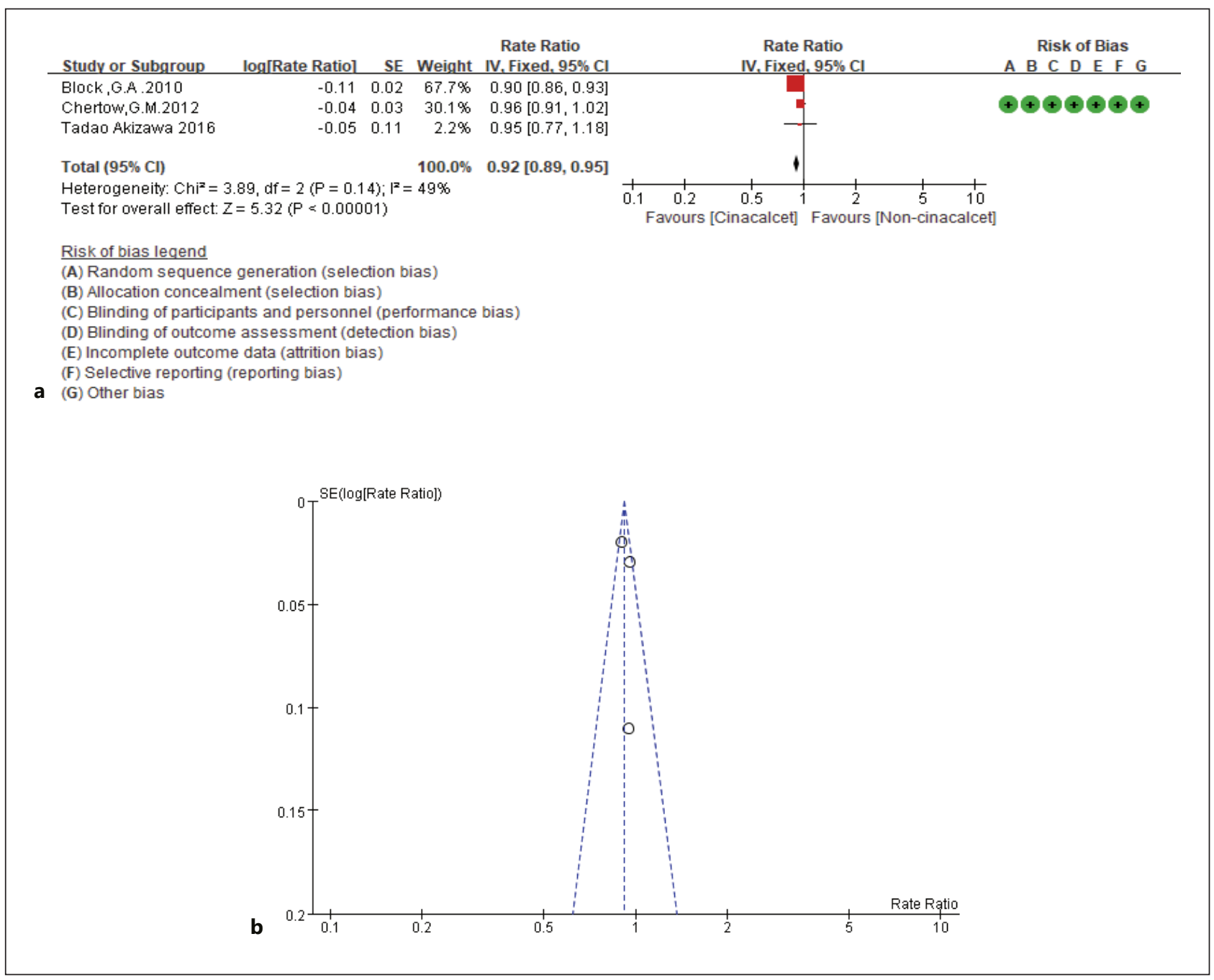

Fig. 3. a Forest plot of cardiovascular mortality in the cinacalcet group versus the no-cinacalcet control group. b Funnel plot to assess publication bias in effect estimates in cardiovascular mortality.

26] with 30,748 participants fulfilled the eligibility criteria and were included in the metaanalysis (Fig. 1; Table 2). All 14 trials included patients with stage 5 CKD who were under dialysis treatment.

All-cause mortality. We included 13 trials (including 38,219 participants) that either reported all-cause mortality or the all-cause mortality could be calculated from the data reported in 2 groups. Our results indicated that cinacalcet reduced all-cause mortality compared with placebo or no cinacalcet treatment in patients with SHPT caused by CKD (RR $0.91,95 \%$ CI $0.89-0.94, p<0.001)$ as determined using a fixed effects model $\left(I^{2}=0 \%, p=0.81\right.$; Fig. 2a, b).

Cardiovascular mortality. Only 3 trials (including 32,309 participants) that either reported cardiovascular mortality or the all-cause mortality could be calculated from the data reported were included in our meta-analysis. The results showed that cinacalcet treatment may reduce cardiovascular mortality compared with no cinacalcet treatment in patients with SHPT caused by CKD (RR 0.92, 95\% CI 0.89-0.95, $p<0.001$ ) as determined using a fixed effects model $\left(I^{2}=49 \%, p=0.14\right.$; Fig. $\left.3 \mathrm{a}, \mathrm{b}\right)$. 


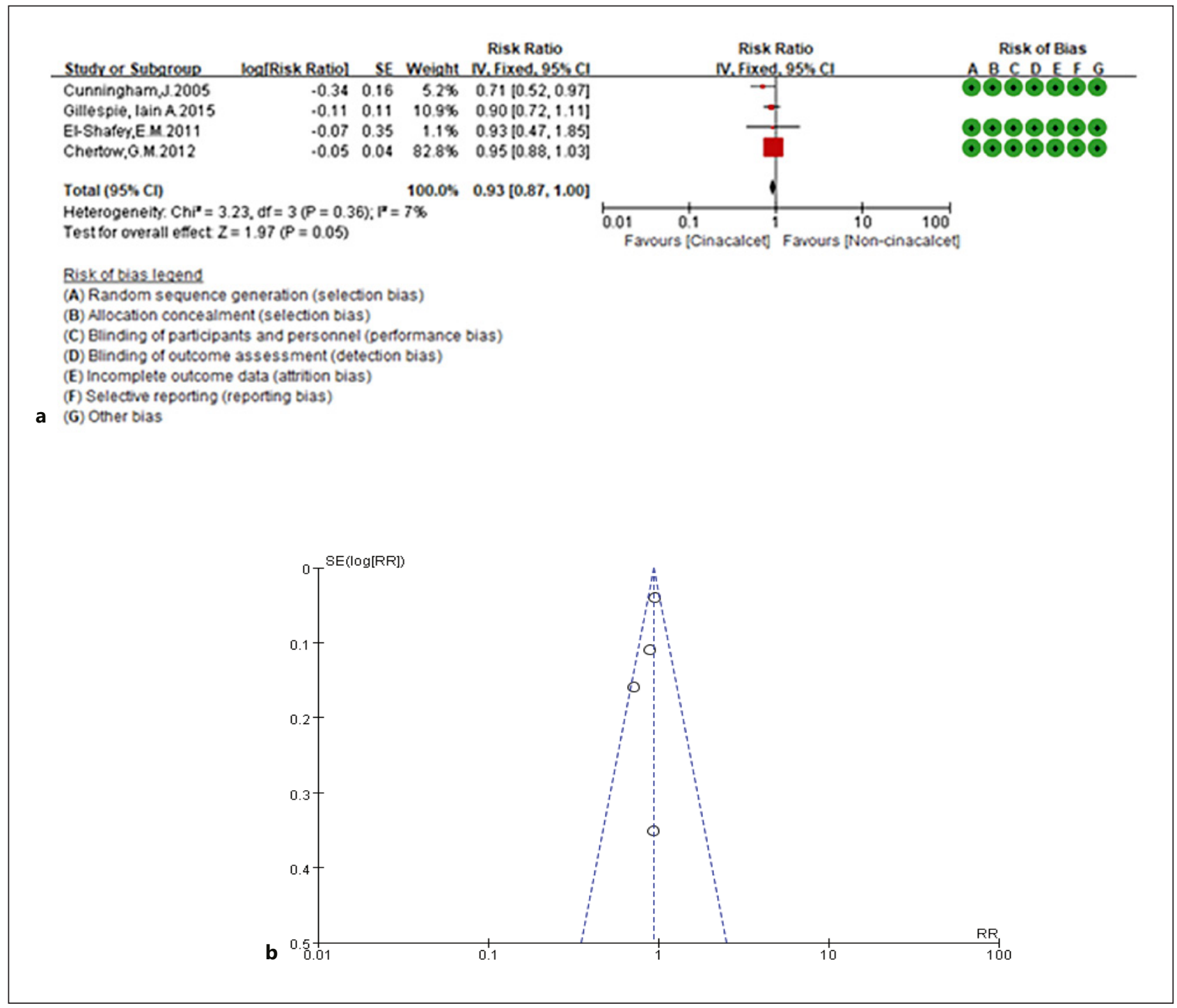

Fig. 4. a Forest plot on fracture incidence in the cinacalcet group versus the non-cinacalcet control group. b Funnel plot to assess publication bias in effect estimates in fracture incidence.

Fracture incidence. Four trials (including 7,471 participants) that either reported the incidence of fractures or the incidence of fractures could be calculated from the data reported were included in our meta-analysis. The results showed that cinacalcet did not significantly reduce the incidence of fractures (RR $0.93,95 \%$ CI $0.87-1.00, p=0.05$ ) as determined using a fixed effects model $\left(I^{2}=7 \%, p=0.36\right.$; Fig. $\left.4 \mathrm{a}, \mathrm{b}\right)$.

\section{Discussion}

Although there were several published meta-analyses of RCTs examining the effects of cinacalcet on CKD-MBD, cardiovascular mortality, and all-cause mortality, this is the first meta-analysis that included RCTs and high-quality prospective observational studies to evaluate the long-term treatment effects of cinacalcet on cardiovascular mortality, all-cause 
Zu et al.: Cinacalcet Treatment Improves All-Cause and Cardiovascular Survival in Dialysis Patients

mortality, and fracture incidence. To our knowledge, this meta-analysis of 10 RCTs and 3 prospective observational studies of patients receiving hemodialysis or peritoneal dialysis is the largest, with 36,283 participants, and the results are not in agreement with the results of previous meta-analyses [27, 28]. Previous meta-analyses included only RCTs. Owing to limited time and money, in most RCTs, observation indicators do not include death. Patients were observed only for several months, and there were almost no deaths. However, many meta-analyses discuss all-cause mortality and cardiovascular mortality [29-31]. We selected end-point events (all-cause or cardiovascular mortalities and fracture incidence) and their HR or RR and $95 \% \mathrm{CI}$ as outcomes to report.

Evidence suggests that maintaining CKD-MBD-related mineral metabolic parameters within the K/DOQI target ranges is of significant clinical relevance [32]. Cinacalcet is widely used in clinical practice, and there is an obvious beneficial effect of cinacalcet on serum PTH, calcium, and phosphorus levels. However, it was not clear whether cinacalcet could reduce hospitalizations due to cardiovascular events, cardiovascular mortality, or all-cause mortality compared with standard treatment for CKD. The strength of the observational data suggests that high levels of calcium and phosphorus are associated with mortality. It is tempting to speculate that serum PTH, calcium, and phosphorus reductions may be the proximate cause of a survival benefit [33]. In addition, abnormal calcium and phosphorus levels in the blood and tissues are linked to vascular calcification, resulting in cardiovascular diseases. In addition, there was a significant negative correlation between PTH and all-cause mortality. It has been repeatedly confirmed that cinacalcet has a lowering effect on serum FGF-23, a marker of left ventricular hypertrophy and cardiovascular disease in the CKD population [34-36]. Therefore, in theory, improvement in the above indicators will help reduce cardiovascular mortality or all-cause mortality. In addition, some long-term high-quality prospective observational studies found that the prescription of cinacalcet to hemodialysis patients with SHPT who received traditional treatment (no-cinacalcet control group) was associated with significant reductions in all-cause and cardiovascular mortality. Our meta-analysis included evidence from both RCTs and observational studies on the effects of cinacalcet on all-cause and CV-related mortality, showing that cinacalcet may be associated with a decreased risk of all-cause $(0.91,95 \%$ CI $0.89-0.94)$ and CV-related mortality $(0.92,95 \%$ CI 0.89-0.95) among patients receiving maintenance dialysis. Because these results are observational and cannot establish a cause-and-effect relationship, they are not currently used in conventional therapy.

Bone remodeling is dependent on the dynamic balance between bone formation and bone resorption. Excessively high levels of serum PTH lead to an imbalance of these 2 processes and affect bone and mineral metabolism [37, 38]. El-Shafey et al. [19] reported that cinacalcet treatment increased the proximal femur BMD in patients with SHPT. In contrast, when SHPT was not effectively treated, there was a progressive loss of the proximal femur BMD. Cinacalcet treatment for 36 weeks not only halted bone loss but also resulted in a $2 \%$ increase in mineralized bone in the proximal femur; however, cinacalcet did not stop the bone loss in the lumbar spine. Some studies have demonstrated that cinacalcet ameliorated high bone turnover and increased femoral cortical bone mineral density and cortical bone strength [39]. These data suggest that cinacalcet may protect cortical bone in patients with SHPT. However, a positive result of reducing the incidence of fractures was not obtained from RCTs. Our meta-analysis results of improving fracture incidence are close to significant.

Previous studies including RCTs, meta-analyses, and observational studies found that the use of cinacalcet was correlated with a relatively high risk of adverse events, including hypocalcemia, nausea, vomiting, and diarrhea. Some findings did not indicate apparent benefits of cinacalcet therapy on mortality, which may preclude the use of cinacalcet as the first-line therapy in CKD patients with uncontrolled hyperparathyroidism. Research by Wang et al. [27] showed that the absolute risk of hypocalcemia, nausea, vomiting, and diarrhea increased 


\section{Kidney \\ Blood Pressure Research}

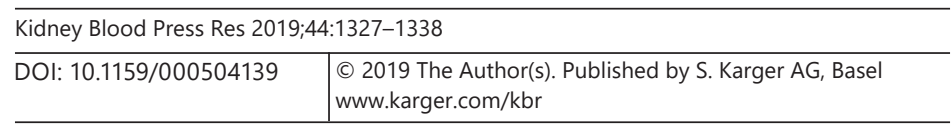

Zu et al.: Cinacalcet Treatment Improves All-Cause and Cardiovascular Survival in Dialysis Patients

by $10.31,13.16,10.57$, and $2.01 \%$ respectively, compared with placebo or no treatment. It is also not clear whether cinacalcet affects all-cause mortality and cardiovascular mortality. These findings limited the prescription of cinacalcet by clinicians. However, in some prospective and retrospective studies, cinacalcet-induced hypocalcemia was of short duration and usually self-limited. Importantly, hypocalcemia-induced serious adverse events were very rare [40]. Accordingly, the revised 2017 KDIGO CKD-MBD guideline states that mild and asymptomatic hypocalcemia can be tolerated in patients on dialysis, especially when associated with cinacalcet treatment, to avoid inappropriate calcium loading in adults [41]. Cinacalcet initiation was more effective in patients with more severe iPTH levels than that in patients with less severe iPTH levels. We combined the results of observational studies and RCTs and found that the treatment of SHPT in dialysis with cinacalcet therapy may reduce all-cause mortality and cardiovascular mortality. Clinicians should seriously consider the benefits and harms of cinacalcet versus standard treatment to persistently control elevated PTH levels due to CKD-MBD in dialysis.

In conclusion, our meta-analysis results showed that treatment of SHPT in maintenance dialysis patients with cinacalcet therapy may reduce all-cause mortality and cardiovascular mortality. However, further RCTs investigating the effect of cinacalcet on long-term mortality and fracture incidence are required.

\section{Acknowledgments}

This work was supported by the National Natural Science Foundation of China (81670673) and the Beijing Natural Science Foundation (7182060).

\section{Disclosure Statement}

The authors had full access to all the data in the study and had the final responsibility for the decision to submit to publication. The results presented in this paper have not been published previously in whole or part. The authors declare no conflicts of interest.

\section{References}

1 Nugent RA, Fathima SF, Feigl AB, Chyung D. The burden of chronic kidney disease on developing nations: a 21st century challenge in global health. Nephron Clin Pract. 2011;118(3):c269-77.

2 Moe S, Drüeke T, Cunningham J, Goodman W, Martin K, Olgaard K, et al.; Kidney Disease: Improving Global Outcomes (KDIGO). Definition, evaluation, and classification of renal osteodystrophy: a position statement from Kidney Disease: Improving Global Outcomes (KDIGO). Kidney Int. 2006 Jun;69(11):1945-53.

3 Nemeth EF. Calcimimetic and calcilytic drugs: just for parathyroid cells? Cell Calcium. 2004 Mar;35(3):283-9.

4 Brown EM, Hebert SC. The First Annual Bayard D. Catherwood Memorial Lecture. Ca2+-receptor-mediated regulation of parathyroid and renal function. Am J Med Sci. 1996 Sep;312(3):99-109.

5 Lindberg JS. Calcimimetics: a new tool for management of hyperparathyroidism and renal osteodystrophy in patients with chronic kidney disease. Kidney Int Suppl. 2005 Jun;67(95):S33-6.

6 Lindberg JS, Culleton B, Wong G, Borah MF, Clark RV, Shapiro WB, et al. Cinacalcet HCl, an oral calcimimetic agent for the treatment of secondary hyperparathyroidism in hemodialysis and peritoneal dialysis: a randomized, double-blind, multicenter study. J Am Soc Nephrol. 2005 Mar;16(3):800-7.

7 Eckardt KU, Kasiske BL. Kidney disease: improving global outcomes. Nat Rev Nephrol. 2009 Nov;5(11):650-7.

8 Martin KJ, González EA. Metabolic bone disease in chronic kidney disease. J Am Soc Nephrol. 2007 Mar;18(3): 875-85.

9 Chertow GM, Block GA, Correa-Rotter R, Drüeke TB, Floege J, Goodman WG, et al.; EVOLVE Trial Investigators. Effect of cinacalcet on cardiovascular disease in patients undergoing dialysis. N Engl J Med. 2012 Dec;367(26): 2482-94. 


\begin{tabular}{l|l}
\hline \multicolumn{2}{l}{ Kidney Blood Press Res 2019;44:1327-1338 } \\
\hline DOI: 10.1159/000504139 & $\begin{array}{l}\text { (c) 2019 The Author(s). Published by S. Karger AG, Basel } \\
\text { www.karger.com/kbr }\end{array}$ \\
\hline
\end{tabular}

Zu et al.: Cinacalcet Treatment Improves All-Cause and Cardiovascular Survival in Dialysis Patients

10 Cunningham J, Danese M, Olson K, Klassen P, Chertow GM. Effects of the calcimimetic cinacalcet HCl on cardiovascular disease, fracture, and health-related quality of life in secondary hyperparathyroidism. Kidney Int. 2005 Oct; 68(4):1793-800.

11 Lozano-Ortega G, Waser N, Bensink ME, Goring S, Bennett H, Block GA, et al. Effects of calcimimetics on longterm outcomes in dialysis patients: literature review and Bayesian meta-analysis. J Comp Eff Res. 2018 Jul; 7(7):693-707.

12 Block GA, Martin KJ, de Francisco AL, Turner SA, Avram MM, Suranyi MG, et al. Cinacalcet for secondary hyperparathyroidism in patients receiving hemodialysis. N Engl J Med. 2004 Apr;350(15):1516-25.

13 Gillespie IA, Floege J, Gioni I, Drueke TB, de Francisco AL, Anker SD, et al. Propensity score matching and persistence correction to reduce bias in comparative effectiveness: the effect of cinacalcet use on all-cause mortality. Pharmacoepidemiol Drug Saf. 2015 Jul;24(7):738-47.

14 Higgins JP, Altman DG, Gotzsche PC, Juni P, Moher D, Oxman AD, et al. The Cochrane Collaboration's tool for assessing risk of bias in randomised trials. BMJ. 2011 Oct;343:d5928.

15 Liberati A, Altman DG, Tetzlaff J, Mulrow C, Gotzsche PC, Ioannidis JP, et al. The PRISMA statement for reporting systematic reviews and meta-analyses of studies that evaluate healthcare interventions: explanation and elaboration. BMJ. 2009 Jul;339:b2700.

16 Stroup DF, Berlin JA, Morton SC, Olkin I, Williamson GD, Rennie D, et al. Meta-analysis of observational studies in epidemiology: a proposal for reporting. Meta-analysis Of Observational Studies in Epidemiology (MOOSE) group. JAMA. 2000 Apr;283(15):2008-12.

17 Higgins JP, Thompson SG, Deeks JJ, Altman DG. Measuring inconsistency in meta-analyses. BMJ. 2003 Sep; 327(7414):557-60.

18 Guyatt GH, Oxman AD, Schünemann HJ, Tugwell P, Knottnerus A. GRADE guidelines: a new series of articles in the Journal of Clinical Epidemiology. J Clin Epidemiol. 2011 Apr;64(4):380-2.

19 El-Shafey EM, Alsahow AE, Alsaran K, Sabry AA, Atia M. Cinacalcet hydrochloride therapy for secondary hyperparathyroidism in hemodialysis patients. Ther Apher Dial. 2011 Dec;15(6):547-55.

20 Fishbane S, Shapiro WB, Corry DB, Vicks SL, Roppolo M, Rappaport K, et al. Cinacalcet HCl and concurrent low-dose vitamin $\mathrm{D}$ improves treatment of secondary hyperparathyroidism in dialysis patients compared with vitamin D alone: the ACHIEVE study results. Clin J Am Soc Nephrol. 2008 Nov;3(6):1718-25.

21 Raggi P, Chertow GM, Torres PU, Csiky B, Naso A, Nossuli K, et al.; ADVANCE Study Group. The ADVANCE study: a randomized study to evaluate the effects of cinacalcet plus low-dose vitamin D on vascular calcification in patients on hemodialysis. Nephrol Dial Transplant. 2011 Apr;26(4):1327-39.

22 Ureña-Torres P, Bridges I, Christiano C, Cournoyer SH, Cooper K, Farouk M, et al. Efficacy of cinacalcet with low-dose vitamin D in incident haemodialysis subjects with secondary hyperparathyroidism. Nephrol Dial Transplant. 2013 May;28(5):1241-54.

23 Wetmore JB, Gurevich K, Sprague S, Da Roza G, Buerkert J, Reiner M, et al. A Randomized Trial of Cinacalcet versus Vitamin D Analogs as Monotherapy in Secondary Hyperparathyroidism (PARADIGM). Clin J Am Soc Nephrol. 2015 Jun;10(6):1031-40.

24 Malluche HH, Monier-Faugere MC, Wang G, Frazã O JM, Charytan C, Coburn JW, et al. An assessment of cinacalcet $\mathrm{HCl}$ effects on bone histology in dialysis patients with secondary hyperparathyroidism. Clin Nephrol. 2008 Apr;69(4):269-78.

25 Block GA, Zaun D, Smits G, Persky M, Brillhart S, Nieman K, et al. Cinacalcet hydrochloride treatment significantly improves all-cause and cardiovascular survival in a large cohort of hemodialysis patients. Kidney Int. 2010 Sep;78(6):578-89.

26 Akizawa T, Kurita N, Mizobuchi M, Fukagawa M, Onishi Y, Yamaguchi T, et al. PTH-dependence of the effectiveness of cinacalcet in hemodialysis patients with secondary hyperparathyroidism. Sci Rep. 2016 Apr;6: 19612.

27 Wang G, Liu H, Wang C, Ji X, Gu W, Mu Y. Cinacalcet versus Placebo for secondary hyperparathyroidism in chronic kidney disease patients: a meta-analysis of randomized controlled trials and trial sequential analysis. Sci Rep. 2018 Feb;8(1):3111.

28 Sekercioglu N, Busse JW, Sekercioglu MF, Agarwal A, Shaikh S, Lopes LC, et al. Cinacalcet versus standard treatment for chronic kidney disease: a systematic review and meta-analysis. Ren Fail. 2016 Jul;38(6):857-74.

29 Goodman WG, Hladik GA, Turner SA, Blaisdell PW, Goodkin DA, Liu W, et al. The Calcimimetic agent AMG 073 lowers plasma parathyroid hormone levels in hemodialysis patients with secondary hyperparathyroidism. J Am Soc Nephrol. 2002 Apr;13(4):1017-24.

30 Fukagawa M, Yumita S, Akizawa T, Uchida E, Tsukamoto Y, Iwasaki M, et al.; KRN1493 study group. Cinacalcet (KRN1493) effectively decreases the serum intact PTH level with favorable control of the serum phosphorus and calcium levels in Japanese dialysis patients. Nephrol Dial Transplant. 2008 Jan;23(1): 328-35.

31 Kim HJ, Kim H, Shin N, Na KY, Kim YL, Kim D, et al. Cinacalcet lowering of serum fibroblast growth factor-23 concentration may be independent from serum Ca, P, PTH and dose of active vitamin D in peritoneal dialysis patients: a randomized controlled study. BMC Nephrol. 2013 May; 14:112.

32 National Kidney Foundation. K/DOQI clinical practice guidelines for bone metabolism and disease in chronic kidney disease. Am J Kidney Dis. 2003 Oct;42(4 Suppl 3):S1-201.

33 Danese MD, Belozeroff V, Smirnakis K, Rothman KJ. Consistent control of mineral and bone disorder in incident hemodialysis patients. Clin J Am Soc Nephrol. 2008 Sep;3(5):1423-9. 


\begin{tabular}{l|l}
\hline DOI: 10.1159/000504139 & $\begin{array}{l}\text { (c) } 2019 \text { The Author(s). Published by S. Karger AG, Basel } \\
\text { www.karger.com/kbr }\end{array}$ \\
\hline
\end{tabular}

Zu et al.: Cinacalcet Treatment Improves All-Cause and Cardiovascular Survival in Dialysis Patients

34 Moe SM, Chen NX. Pathophysiology of vascular calcification in chronic kidney disease. Circ Res. 2004 Sep; 95(6):560-7.

35 Speer MY, Giachelli CM. Regulation of cardiovascular calcification. Cardiovasc Pathol. 2004 Mar-Apr;13(2): 63-70.

36 Isakova T, Xie H, Yang W, Xie D, Anderson AH, Scialla J, et al. Fibroblast growth factor 23 and risks of mortality and end-stage renal disease in patients with chronic kidney disease. JAMA. 2011 Jun 15;305(23):2432-9.

37 Colloton M, Shatzen E, Miller G, Stehman-Breen C, Wada M, Lacey D, et al. Cinacalcet HCl attenuates parathyroid hyperplasia in a rat model of secondary hyperparathyroidism. Kidney Int. 2005 Feb;67(2):467-76.

38 Malluche HH, Mawad H, Monier-Faugere MC. The importance of bone health in end-stage renal disease: out of the frying pan, into the fire? Nephrol Dial Transplant. 2004 Mar;19 Suppl 1:19-13.

39 Fukuhara S, Akizawa T, Fukagawa M, Onishi Y, Yamaguchi T, Hasegawa T, et al. Mineral and bone disorders outcomes study for Japanese chronic kidney disease stage 5D patients: rationale and study design. Ther Apher Dial. 2011 Apr;15(2):169-75.

40 Floege J, Tsirtsonis K, Iles J, Drueke TB, Chertow GM, Parfrey P. Incidence, predictors and therapeutic consequences of hypocalcemia in patients treated with cinacalcet in the EVOLVE trial. Kidney Int. 2018 Jun;93(6): 1475-82.

41 Ketteler M, Block GA, Evenepoel P, Fukagawa M, Herzog CA, McCann L, et al. Executive summary of the 2017 KDIGO Chronic Kidney Disease-Mineral and Bone Disorder (CKD-MBD) Guideline Update: what's changed and why it matters. Kidney Int. 2017 Jul; 92(1):26-36. 